\title{
REVIEW
}

\section{Integrins and their ligands in rheumatoid arthritis}

Torsten Lowin* and Rainer H Straub

\begin{abstract}
Integrins play an important role in cell adhesion to the extracellular matrix and other cells. Upon ligand binding, signaling is initiated and several intracellular pathways are activated. This leads to a wide variety of effects, depending on cell type. Integrin activation has been linked to proliferation, secretion of matrixdegrading enzymes, cytokine production, migration, and invasion. Dysregulated integrin expression is often found in malignant disease. Tumors use integrins to evade apoptosis or metastasize, indicating that integrin signaling has to be tightly controlled. During the course of rheumatoid arthritis, the synovial tissue is infiltrated by immune cells that secrete large amounts of cytokines. This pro-inflammatory milieu leads to an upregulation of integrin receptors and their ligands in the synovial tissue. As a consequence, integrin signaling is enhanced, leading to enhanced production of matrix-degrading enzymes and cytokines. Furthermore, in analogy to invading tumors, synovial fibroblasts start invading and degrading cartilage, thereby generating extracellular matrix debris that can further activate integrins.
\end{abstract}

Integrins are a large group of transmembrane proteins that anchor the cell to the extracellular matrix (ECM) or other cells. Upon binding, integrins remodel the ECM by inducing the expression of certain proteases. Integrins control cellular proliferation, migration, and invasion. Whereas in some cases integrins can mediate these effects on their own, they usually signal in the context of growth factor or cytokine receptors [1]. Ligand binding to integrin controls adhesion-dependent proliferation, whereas unligated integrins enhance apoptotic pathways [2]. This is one reason why dysregulated integrin expression or downstream signaling pathways can be observed in almost

*Correspondence: torsten.lowin@klinik.uni-regensburg.de Laboratory of Experimental Rheumatology and Neuroendocrine Immunology, Department of Internal Medicine, University Hospital Regensburg, Franz-Josef Strauß Allee 11, 93053 Regensburg, Germany all forms of cancer, and integrin levels often determine the aggressiveness or propensity to metastasize.

Migration, invasion, and proliferation of synovial cells are major problems in rheumatoid arthritis (RA) [3]. This debilitating disease is characterized by an inflamed synovial tissue with a massive influx of immune cells and an inflated synovial lining consisting mainly of synovial fibroblasts (SFs) and macrophages that adhere to the adjacent ECM. These SFs are highly proliferative and contribute significantly to cartilage and bone destruction. In some aspects, they can be considered 'tumor-like' as SFs are resistant to many apoptotic pathways, show increased proliferation, and produce high amounts of matrix metalloproteinases (MMPs) because of dysregulation of the transcription factors AP-1, p53, and nuclear factor-kappa-B (NF-kB) [4]. Fibroblasts, macrophages, but also endothelial cells in synovial tissue show high levels of integrin expression [5]. In RA, not only integrins but also their ligands (for example, fibronectin and collagen as well as their degradation products) are upregulated [6]. This 'overstimulation' of cells by integrinmediated signals increases basal secretion of proinflammatory cytokines like interleukin-6 (IL-6) and IL-8 but also levels of various MMPs [7-9].

This review introduces the predominant integrins expressed on synovial cells along with their binding partners and demonstrates their significance in disease progression. We focused mainly on the $\beta 1, \beta 3$, and $\beta 5$ integrins because altered levels of their ligands and respective integrins themselves are one major factor that fuels the destruction of cartilage in RA. Although integrins influence RA significantly, only a few reviews that address integrins in RA have been published. A Medline search for 'integrins' and 'arthritis' yielded 108 (compared with 'integrins' and 'cancer' with over 1,200 reviews) reviews, most of which outlined single integrins or cell types. Two reviews in 1993 and 1995 - by Postigo and colleagues [10] and Liao and Haynes [11], respectively - were the last comprehensive articles describing the influence of integrins on the pathogenesis of RA. Both articles, however, failed to describe the intricacies in integrin biology, from the generation of integrin ligands from the ECM to the subsequent triggering of integrin signaling cascades that finally lead to the generation of pro-inflammatory factors and cartilage 
degradation. To our knowledge, this is the first review that demonstrates the whole integrin machinery in arthritis, including ligands, receptors, and effector cells.

\section{Integrin biology}

A cell needs to sense the composition of the extracellular environment to elicit appropriate responses. Integrins, a family of heterodimeric proteins, serve as sensors of the ECM. Integrins consist of an $\alpha$ subunit that determines ligand specificity and a $\beta$ subunit that initiates intracellular signaling events. Whereas most integrins bind almost exclusively to ECM molecules, $\beta 2$ heterodimers bind other adhesion molecules, mediate cell-cell contacts, and are important components of the immune system. In contrast to the $\beta 2$ integrin, which is expressed only on immune cells, other $\beta$ subunits have a much more diverse expression. In the synovium, fibroblasts, macrophages, and endothelial cells are just a few examples of cells that express the $\beta 1, \beta 3$, and $\beta 5$ subunits. The ECM-binding integrins mediate cellular processes such as adhesion, migration, tissue invasion, and chemotaxis but also cytokine and MMP production.

Within the protein sequences of fibronectin, collagen, and vitronectin, integrins bind to arginine-glycineaspartic acid (RGD) motives. Interaction with the RGD sequence is an important switch for integrin activation and subsequent cellular changes. Integrins can transduce signals from outside the cell to the interior and vice versa, allowing the cell to respond dynamically to a wide variety of stimuli. Growth factors, hormones, and cytokines as well as the composition of the ECM are able to modulate integrin signaling and affinity [12]. When integrins are activated by ligands, several signaling events are initiated. Besides the focal adhesion kinase (FAK), the mitogenactivated protein kinase (MAPK) pathway, the phosphatidylinositol 3-kinase (PI3K), and c-Jun N-terminal kinases (JNKs) are activated [13]. Growth factors and cytokines employ the same signaling pathways as integrins, demonstrating an intricate interplay between the two. Integrins are linked to the cytoskeleton by coupling to adaptor proteins such as vinculin and talin. Therefore, changes in integrin activation lead to changes in the actin cytoskeleton, a prerequisite for adhesion, migration, and invasion on ECMs.

\section{Integrin knockouts and related pathologies}

Given that various integrin combinations recognize the same ECM molecule (for example, fibronectin is recognized by $\alpha 4 \beta 1, \alpha 5 \beta 1, \alpha \nu \beta 1, \alpha \nu \beta 3$, and $\alpha 3 \beta 1$ and weakly by others), one would expect some redundancy in integrin function. In fact, this is the case when collagen-binding integrin subunits $\alpha 1$ or $\alpha 2$ are knocked out. When $\alpha 1$ is knocked out, corresponding mice show decreased vascularization, and isolated endothelial cells from these animals show reduced proliferation whereas $\alpha 2$ knockouts demonstrate only a subtle phenotype. The picture is different when regarding the knockout of fibronectinbinding integrin subunits. Knockout of either integrin $\alpha 3$, $\alpha 4, \alpha 5$ or $\alpha v$ is lethal during embryogenesis and respective mice die shortly after birth. Also, the fibronectinbinding integrins have a more tissue-specific distribution than collagen-binding integrins, making target cells more susceptible to specific knockouts.

Besides having a role in development, integrin mutations lead to a variety of other defects. Leukocyte adhesion deficiency type 1 (LAD-1) is an autosomal recessive disorder based on point mutations within the $\beta 2$ integrin, resulting in either its absence on the cell surface or the inability to associate with the corresponding $\alpha$ subunit. This leads to reoccurring bacterial infections, suggesting an impaired function of macrophages and neutrophils.

Another autosomal recessive (although an autoimmune form exists) disease is the Glanzmann thrombasthenia, in which the platelet integrin $\alpha \operatorname{IIb} \beta 3$ fails to bind to fibrinogen and fibrin. This step is important to form blood clots after injury, and failure to do so leads to prolonged bleeding.

Integrins are also involved in the generation and perpetuation of cancer. In several tumors, expression of specific integrin subunits is associated with malignancy. In melanoma, increased expression of $\alpha v \beta 3$ and $\alpha 5 \beta 1$ promotes vertical growth and lymph node metastasis. In non-small-cell lung carcinoma, high $\alpha 5 \beta 1$ levels predict decreased patient survival. In general, alterations in integrin activation or expression promote tumor growth and metastasis. However, in some cases (for example, $\alpha 2 \beta 1$ in breast cancer), integrins can be protective by decreasing cell dissemination [14]. This integrin increases differentiation of epithelium and enhances ECM binding, thus decreasing migration.

\section{Integrins in synovial tissue}

When the integrin distribution in the synovial tissue is analyzed, several factors need to be considered. First, integrin expression and function differ depending on cell type. Second, the location of the cell is important as expression levels differ at the synovial lining compared with the sublining. Every cell in the synovial tissue expresses a specific subset of integrins, depending on lineage and origin. Several groups have investigated synovial tissue samples and isolated cells to study integrin expression. The integrin subunit $\beta 1$ is most widely expressed since it can be found on macrophages, lymphocytes, endothelial cells, and fibroblasts $[5,15,16]$. The fibronectin receptor $\alpha 5 \beta 1$ and the laminin receptor $\alpha 3 \beta 1$ were also found on every synovial cell type, whereas $\alpha 4 \beta 1$, which besides fibronectin also binds VCAM-1, was found only on lymphocytes. Endothelial cells and 
fibroblasts also express the collagen receptor $\alpha 1 \beta 1$ and the vitronectin receptor $\alpha v \beta 3$ [17]. In synovial tissue, integrin expression also depends on the location of the particular cell. Expression of most integrins is similar throughout the synovial tissue but is different at the synovial lining layer [5]. This is the area where synovial cells adhere to cartilage and activated fibroblasts and macrophages degrade ECM and invade cartilage. The laminin receptor $\alpha 6 \beta 1$ is expressed by fibroblasts at the lining layer but not by macrophages, which usually express this receptor [18]. At the synovial lining, we found an increase in $\alpha 5, \alpha v$, and $\beta 1$ integrin levels compared with the sublining area. This clearly indicates that increased integrin expression is associated with a more 'destructive' phenotype of the corresponding cell.

Figure 1 depicts the integrin-ligand interactions and their consequences (for example, proliferation and secretion of pro-inflammatory mediators) described in the next three sections. The most important cell types (fibroblasts, endothelium, $\mathrm{T}$ cells, B cells, macrophages, and Th17 cells), including their individual reaction to integrin engagement, are depicted (Figure 1). The individual integrin levels on each cell type are presented in Table 1. This table reflects our own observations of tissue samples stained for individual integrins and flow cytometric analyses as well as literature data.

\section{The collagen-binding integrins $\alpha 1 \beta 1$ and $\alpha_{2} \beta 1$}

Collagens are widely expressed in synovial tissue, and, during the course of RA, a significant proportion of patients develop antibodies against at least one collagen subtype. The collagens II, IX, X, and XI are restricted to cartilage, whereas all other forms can also be found within the ECM. The classical collagen-binding integrins rely on the triple-helical structure for binding [19]. However, if collagen is proteolytically degraded, RGD sequences are exposed and other RGD-binding integrins can bind. In this case, $\alpha \nu \beta 3$, $\alpha \operatorname{IIb} \beta 3$, and $\alpha 5 \beta 1$ can be activated by collagen-derived RGD sequences [20].

Four different integrin combinations recognize native collagen: $\alpha 1 \beta 1, \alpha 2 \beta 1, \alpha 10 \beta 1$, and $\alpha 11 \beta 1$. Integrin $\alpha 10 \beta 1$ is important for chondrogenesis, whereas $\alpha 11 \beta 1$ has a role in the recognition and organization of interstitial collagen matrices during development. During the course of arthritis, only $\alpha 1 \beta 1$ and a $2 \beta 1$ seem to play a role; this is because these are the two major collagen-binding integrins in the synovial tissue. Although all of the abovementioned subunits bind to collagen, their respective specificities differ for each collagen subtype; for example, integrin $\alpha 1$ preferentially binds collagen type IV.

The integrin $\alpha 1$ was found to be expressed at the synovial lining and in blood vessels of arthritic joints. Interestingly, expression was increased compared with osteoarthritis and trauma controls, suggesting that the pro-inflammatory milieu in RA promotes integrin $\alpha 1$ synthesis [21]. However, our group also found an increase of this adhesion receptor on synovial fibroblasts after treatment with cortisol [5]. An increase of $\alpha 1$ - in analogy to integrin $\alpha 5$, which is also upregulated by cortisol potentially increases adhesion to collagen. Owing to cortisol action, the increase in integrin expression is uncoupled from integrin signaling because intracellular signaling pathways are silenced by glucocorticoid treatment.

Lymphocytes also express integrin $\alpha 1$, but only synovial lymphocytes show increased expression of this receptor [22]. One important modulator for $\alpha 1$ levels is tumor necrosis factor (TNF) because anti-TNF therapy reduces the number of $\alpha 1$-positive effector memory $\mathrm{T}$ cells that contribute significantly to inflammation by producing large amounts of interferon-gamma (IFN- $\gamma$ ). Besides adhesion to collagen, $\alpha 1$ mediates several other effects, depending on cell type. On endothelial cells, vascular endothelial growth factor (VEGF) strongly upregulates $\alpha 1$, which is a prerequisite for the formation of new blood vessels. Inhibition of the integrin/collagen binding by specific antibodies reduced the formation of new blood vessels but did not alter pre-existing vasculature [23]. As angiogenesis is important for the progression of arthritis, its inhibition could be an attractive target in the therapy of RA. Engagement of integrin $\alpha 1$ on macrophages and $T$ cells stimulates migration and cytokine production [24]. The role of $\alpha 1$ on synovial fibroblasts has not been elucidated yet, but studies from dermal fibroblasts suggest that $\alpha 1$ controls collagen synthesis, migration, and proliferation on collagen substrates. In a murine anti-collagen II antibody-induced arthritis model, inhibition of integrin $\alpha 1$ reduced cartilage degradation and leukocyte infiltration, demonstrating an important role for this integrin in inflammation [25].

Integrin $\alpha 2 \beta 1$ has functions similar to those of $\alpha 1 \beta 1$ (for example, VEGF-induced proliferation of endothelial cells) but is much weaker in expression in the synovial tissue. Ligation of $\alpha 2 \beta 1$ by collagen type I or II, but not collagen type IV, augmented IFN- $\gamma$ production in T cells. In contrast to $\mathrm{CD} 4^{+}$and $\mathrm{CD} 8^{+} \mathrm{T}$ cells, which upregulate $\alpha 1 \beta 1$ after activation, Th17 cells upregulate $\alpha 2 \beta 1$, which is thought to be a co-stimulatory molecule necessary for IL-17 production [26]. In a model of experimental autoimmune encephalomyelitis, an antibody against $\alpha 2$ suppressed clinical signs and central nervous system inflammation because of reduced extravasation of immune cells. Since leukocyte infiltrates also fuel synovial inflammation, one would expect that $\alpha 2$ inhibition might also be beneficial in the joint.

\section{Laminin-binding integrins $\alpha 3 \beta 1$ and $\alpha 6 \beta 1$}

Laminins are a major component of the basal lamina and usually are secreted by epithelial cells, endothelium, and 


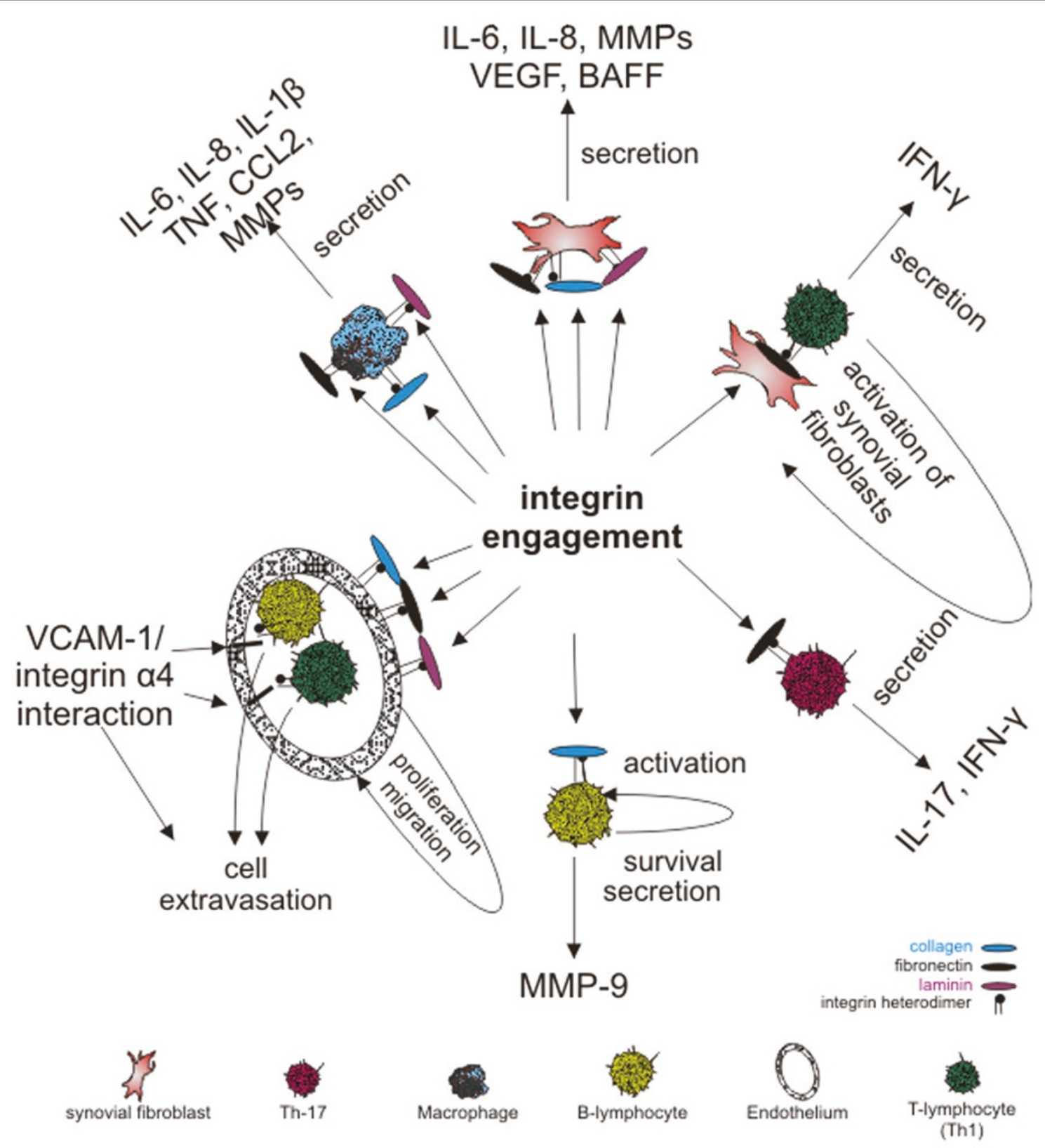

Figure 1. Engagement with fibronectin-, laminin-, or collagen-binding integrins leads to the production and secretion of pro-inflammatory factors. Binding is indicated by the arrows. Cell type, ligand (for example, fibronectin or fibronectin-derived peptides), ligand occupation, and integrin expression level determine the cellular response. BAFF, B-lymphocyte activating factor; CCL2, chemokine (C-C motif) ligand 2; IFN- $\gamma$, interferon-gamma; IL, interleukin; MMP, matrix metalloproteinase; TNF, tumor necrosis factor; VCAM-1, vascular cell adhesion protein 1; VEGF, vascular endothelial growth factor.

organogenic fibroblasts. Laminin is a trimeric molecule consisting of an $\alpha$-chain, a $\beta$-chain, and a $\gamma$-chain that can combine to form 15 different laminin isoforms [27]. Several integrin heterodimers recognize laminin as a ligand, but the affinity for each laminin subtype differs. Laminin binds to integrins $\alpha 3 \beta 1, \alpha 6 \beta 1, \alpha 7 \beta 1$, and $\alpha 6 \beta 4$, but only the first two are present in the synovial tissue. In the synovial tissue, $\alpha 6 \beta 1$ is strongly expressed in the synovial lining layer by fibroblasts, whereas $\alpha 3 \beta 1$ is also expressed by lymphocytes, macrophages, and endothelial cells [18]. Not only the number of laminin receptors but also the production of laminin itself is upregulated in the synovial lining. Besides endothelial cells, SFs produce and secrete laminin, and its deposition at the synovial lining 
Table 1. Surface integrin levels of the predominant cell types in the rheumatoid arthritis synovium

\begin{tabular}{lcccccccccccc}
\hline & a1 & a2 & a3 & a 4 & a5 & a6 & av & $\beta 1$ & $\beta 2$ & $\beta 3$ & $\beta 5$ \\
\hline Synovial fibroblast & ++ & - & $++)$ & - & +++ & + & +++ & +++ & & - & ++ & $?$ \\
Macrophage & + & + & + & +++ & +++ & $(+)$ & +++ & +++ & ++ & +++ & + \\
Endothelium & ++ & + & ++ & + & +++ & + & +++ & +++ & & & +++ & + \\
Tlymphocyte (Th1) & ++ & + & ++ & +++ & +++ & + & +++ & +++ & ++ & +++ & + \\
Th17 cell & - & ++ & ++ & ++ & +++ & + & +++ & +++ & ++ & $?$ & $?$ \\
B lymphocyte & ++ & + & ++ & +++ & +++ & + & ++ & +++ & ++ & $?$ & $?$ \\
\hline
\end{tabular}

For most integrins, expression level data in the literature are given as 'high' or 'strong'. This makes it hard to estimate integrin levels on different cell populations. We consulted several publications and, albeit subjectively, categorized integrin expression levels on a range from strong to weak expression (indicated by one, two, or three plus signs). ?, There is no available data in the literature about the expression of respective integrins.

is increased in RA [28]. Along with collagen and fibronectin, laminin promotes adhesion and proliferation of synovial cells and lymphocytes. Furthermore, integrin binding to laminin increases the expression of MMP-3 and MMP-10, which is further augmented when transforming growth factor-beta (TGF- $\beta$ ) is present [29]. Although no studies have focused on the contribution of laminin and its respective integrin receptors on the progression of RA, results from tumor research indicate that upregulation of $\alpha 6 \beta 1$ or $\alpha 3 \beta 1$, at least in most cases, increases cell invasion and metastasis. As a consequence, increased laminin deposition and increased integrin expression on synovial lining cells increase MMP secretion by these cells, resulting in greater joint damage and invasion into cartilage.

\section{Fibronectin-binding integrins $\alpha 4 \beta 1, \alpha 5 \beta 1, \operatorname{av} \beta 3$, and $\mathbf{\alpha v \beta 5}$}

Fibronectin is a large glycoprotein consisting of two monomers linked by disulfide bonds. Owing to alternative splicing, fibronectin exists in 20 different isoforms. The molecule is folded into globular domains, each of which has a different function. In these domains, repeating amino acid sequences form the so-called type I, II, and III repeats. These sequences interact with other proteins; for example, type I repeats bind to fibrin, and regions in type III repeats contain the RGD motive that binds integrins. Fibronectin can be divided, on the basis of solubility, into two classes: soluble plasma fibronectin and insoluble cellular fibronectin, the latter of which is organized into fibrils as a scaffold for the ECM [30]. Fibronectin levels are elevated in cartilage and synovial fluid of patients with osteoarthritis, whereas in RA, fibronectin is highly expressed at the synovial lining and invading pannus [31].

About 12 integrin heterodimers bind to fibronectin but only some combinations are found in the synovial tissue. The classical fibronectin receptor $\alpha 5 \beta 1$ recognizes only fibronectin, whereas $\alpha v \beta 3$ and $\alpha v \beta 5$ also bind to other ECM molecules or cellular proteins. The $\alpha 5$ integrin is expressed by all cells in the synovial tissue, and $\alpha 5$ levels are increased in the synovial lining layer [5]. Lymphocytes have been shown to attach to other synovial cells by binding to the fibronectin coating on their cell surface by $\alpha 4 \beta 1$ and $\alpha 5 \beta 1$ and to some extent by $\alpha v \beta 3$ integrins [32]. Adhesion to fibronectin increases proliferation and survival of chondrocytes and protects synovial cells from Fas-induced apoptosis [33]. In addition, Zeisel and colleagues [34] found an increase of MMP-3 production by SFs after stimulation with a bacterial ligand of $\alpha 5 \beta 1$, suggesting that enhanced integrin ligation increases joint destruction in RA. Matrix-degrading enzymes in synovial fibroblasts are induced by small integrin-binding peptides derived from the degradation of fibronectin and other ECM molecules. Werb and colleagues [7] demonstrated that only fibronectin-derived fragments, but not intact fibronectin, increased the expression of MMP-1 and MMP-3. Besides having a role in cell survival and proliferation, $\alpha 5 \beta 1$ also regulates cytokine and growth factor production. In SFs, ligation of $\alpha 5 \beta 1$ increased synthesis of B-lymphocyte activating factor (BAFF) [35]. Increased BAFF synthesis not only promotes the proliferation of $\mathrm{B}$ cells and immunglobulin classswitching but possibly acts as an autocrine mechanism of SFs to stimulate their own NF- $\mathrm{kB}$ activity. Besides BAFF, direct ligation of $\alpha 5 \beta 1$ induces the expression of NF- $\mathrm{KB}$ controlled genes in fibroblasts and endothelial cells. Most of these genes - like $I L-8$ and heparin-bound epidermal growth factor (HB-EGF) - or endothelin-1 control angiogenesis or inflammation and their enhanced expression aggravate RA.

Naïve and memory $\mathrm{T}$ cells depend on binding of a $4 \beta 1$ and $\alpha 5 \beta 1$ to fibronectin to promote proliferation [32]. In this context, SFs assist in T-cell activation, as T cells bind to the fibronectin coating on their surface and $\alpha 4 \beta 1$ transmits co-stimulatory signals. In RA, infiltrated T cells in the synovial tissue display higher surface levels of $\alpha 4 \beta 1$ compared with peripheral $\mathrm{T}$ cells and localize to sites of tissue injury by binding to proteolytically degraded cartilage fragments. The most important function of $\alpha 4 \beta 1$ on lymphocytes is adhesion to endothelium and subsequent transmigration to sites of injury. 
Unfortunately, extravasation of lymphocytes into the synovial tissue is also the mechanism that further supports the inflammatory process. In RA, endothelium expresses high levels of VCAM-1, which acts as a binding partner for $\alpha 4 \beta 1$. After chemokine-induced activation and clustering of $\alpha 4 \beta 1$, lymphocytes are immobilized on endothelium and transmigrate to sites of inflammation [36]. The important role for integrin $\alpha 4 \beta 1$ in RA is further underlined by a study by Raychaudhuri and colleagues [37], who show that antagonism of this receptor prevents inflammation and MMP production in a murine model of accelerated collagen-induced arthritis.

Heterodimers containing $\alpha \nu$ are another family of fibronectin-binding integrins. A polymorphism in the gene coding for integrin $\alpha v$ is associated with RA in the European Caucasian population [38]. The integrin $\alpha v \beta 3$ binds fibronectin with high affinity and acts in concert with $\alpha 5 \beta 1$ to arrange fibronectin matrices [39]. Besides binding fibronectin, $\alpha \nu \beta 3$ binds vitronectin, osteopontin, and bone sialoprotein with high affinity. This integrin heterodimer is expressed on SFs, endothelial cells, and osteoclasts [40]. Not much is known about the specific role of $\alpha v \beta 3$ on SFs, but studies from other cell systems and from tumor research indicate that this integrin promotes tissue invasion and MMP-2 production. The major function of $\alpha v \beta 3$ (and also of $\alpha v \beta 5$ ) together with vascular endothelial growth factor receptor 2 (VEGF2R) is angiogenesis promotion [41]. Integrin $\alpha v \beta 3$ does so by phosphorylating intracellular residues of the VEGR2R, thereby enhancing signaling of this receptor. Whereas the expression of $\alpha \nu \beta 3$ is low on resting endothelium, levels increase with inflammation and tumor angiogenesis. In RA, the pro-inflammatory milieu promotes angiogenesis, and its inhibition by a small-molecule $\alpha v$ antagonist was efficacious in a rabbit model of arthritis. Whether this is applicable to humans remains to be seen as the development of vitaxin, a monoclonal antibody against $\alpha v \beta 3$, was discontinued in 2004 because the drug lacked efficacy in RA. It could well be that vitaxin is only minimally effective by itself but potentiates the effect of other therapies. This phenomenon is evident with cilengitide, a small-peptide $\alpha v$ antagonist that is being clinically tested against a variety of cancers. This peptide not only inhibits angiogenesis but also increases the permeability of endothelium. This could be exploited to increase the delivery of other drugs [42].

Another cell type that responds to $\alpha \nu$ antagonists is the osteoclast. In RA, osteoclast activity is enhanced because of increased RANKL (receptor activator of NF-KB ligand) expression, and osteoclasts are responsible for bone erosions in RA [43]. The integrin $\alpha v \beta 3$ increases the bone resorptive capacity of osteoclasts by activating several intracellular signaling pathways such as FAK and c-Src. Blockage of this integrin was protective in vitro.

\section{The $\beta 2$ integrins (CD18)}

In contrast to all of the abovementioned integrin heterodimers, $\beta 2$ integrins bind other adhesion receptors and are expressed exclusively on immune cells. The focus of this section is the $\alpha \mathrm{L} \beta 2$ integrin (lymphocyte functionassociated antigen 1, or LFA-1). This is the major integrin of leukocytes and is important for tissue extravasation, chemotaxis, and formation of immunological synapses. The ligand for LFA-1 is intracellular adhesion molecule (ICAM), which is expressed in three different isoforms. ICAM-1 is expressed in the synovial tissue by endothelial cells and fibroblasts, and cell surface levels of this glycoprotein are increased by the action of cytokines like IL-1 $\beta$. As the pro-inflammatory milieu in RA also leads to the upregulation of $\alpha \mathrm{L} \beta 2$ on leukocytes, the net effect is an enhanced influx of immune cells in the synovial tissue, contributing significantly to inflammation. Increased cell-cell contacts between synovial fibroblasts and $\mathrm{T}$ lymphocytes lead to the activation of fibroblasts and result in an increased cytokine production by both T-cells and fibroblasts. This effect was dependent on T-cell adhesion on fibroblasts as T-lymphocyte cell culture supernatants failed to elicit appropriate responses [44]. Furthermore, $\alpha \mathrm{L} \beta 2$ is part of the immunological synapse between antigen-presenting cells and lymphocytes, and disruption of this interaction leads to immune impairments. Owing to its importance, LFA-1 is a suitable target for therapeutic intervention, and inhibition of this integrin by a mononclonal antibody was efficacious in a rabbit model of RA [45]. The positive effect of LFA-1 inhibition was also shown in mice treated with a small-molecule antagonist against this integrin. Animals showed reduced adhesion of T-cells to endothelial cells, reduced T-cell proliferation, and decreased Th1 cytokine production [46]. In humans, efalizumab, a monoclonal antibody against the $\alpha \mathrm{L}$ subunit, was withdrawn from the market as serious side effects occurred because of the immunosuppressive action of the drug [47]. A more feasible approach to dampen the activity of lymphocytes in RA is the inhibition of ICAM-1. Mice deficient in ICAM-1 showed only minor disease activity in a collagen-induced arthritis model, and clinical studies in humans also demonstrated beneficial effects when ICAM-1 was blocked by an anti-ICAM-1 monoclonal antibody in early RA $[48,49]$. Unfortunately, this antibody could not be administered repeatedly, as its mouse origin makes this antibody immunogenic, resulting in side effects such as fever and leukopenia [50].

\section{Conclusions}

During the course of RA, immune cells are recruited into the synovial tissue, where they produce large amounts of pro-inflammatory cytokines and interact with residential fibroblasts and macrophages. The rate of migration of 
immune cells into the synovium is controlled by the expression of ICAM-1 on endothelial cells and integrin $\alpha \mathrm{L} \beta 2$ on immune cells. The interaction of $T$ lymphocytes (Th1 type) with synovial fibroblasts, endothelial cells, and macrophages activates those cells and they start to produce ECM proteins, cytokines, adhesion molecules, and matrix-degrading enzymes. The pro-inflammatory milieu also increases the expression of most integrin subunits. The combination of increased integrin expression, increased ECM deposition, and degradation along with high cytokine and growth factor levels in the synovial tissue make a perfect microenvironment for the perpetuation of RA. The enhanced integrin signaling in RA is probably one cause of the high basal secretion of cytokines from fibroblasts and macrophages. Furthermore, increased integrin ligation enhances growth factor and cytokine signaling and induces the expression of MMPs. MMPs degrade ECM molecules, thereby generating small RGD peptides, which further activate integrins. When all the facts are taken together, it can be said that RA is, at least in part, an integrin-driven disease that can be modulated with specific integrin antagonists.

\section{Abbreviations}

BAFF, B-lymphocyte activating factor; ECM, extracellular matrix; FAK, focal adhesion kinase; ICAM, intracellular adhesion molecule; IFN- $\gamma$, interferongamma; IL, interleukin; LFA-1, lymphocyte function-associated antigen 1; MMP, matrix metalloproteinase; NF-kB, nuclear factor-kappa-B; RA, rheumatoid arthritis; RGD, arginine-glycine-aspartic acid; SF, synovial fibroblast; TNF, tumor necrosis factor; VCAM-1, vascular cell adhesion protein 1; VEGF, vascular endothelial growth factor; VEGF2R, vascular endothelial growth factor receptor 2 .

\section{Competing interests}

The authors declare that they have no competing interests.

Published: 28 October 2011

\section{References}

1. Stupack DG: Integrins as a distinct subtype of dependence receptors. Cell Death Differ 2005, 12:1021-1030.

2. Stupack DG, Puente XS, Boutsaboualoy S, Storgard CM, Cheresh DA: Apoptosis of adherent cells by recruitment of caspase- 8 to unligated integrins. J Cell Biol 2001, 155:459-470.

3. Davis LS: A question of transformation: the synovial fibroblast in rheumatoid arthritis. Am J Pathol 2003, 162:1399-1402.

4. Bartok B, Firestein GS: Fibroblast-like synoviocytes: key effector cells in rheumatoid arthritis. Immunol Rev 2010, 233:233-255.

5. Lowin T, Straub RH, Neumann E, Bosserhoff A, Vogel C, Moissl C, Anders S, Muller-Ladner U, Schedel J: Glucocorticoids increase alpha5 integrin expression and adhesion of synovial fibroblasts but inhibit ERK signaling, migration, and cartilage invasion. Arthritis Rheum 2009, 60:3623-3632.

6. Wollheim FA: Predictors of joint damage in rheumatoid arthritis. APMIS 1996, 104:81-93.

7. Werb Z, Tremble PM, Behrendtsen O, Crowley E, Damsky CH: Signal transduction through the fibronectin receptor induces collagenase and stromelysin gene expression. J Cell Biol 1989, 109:877-889.

8. Sarkissian M, Lafyatis R: Integrin engagement regulates proliferation and collagenase expression of rheumatoid synovial fibroblasts. J Immuno 1999, 162:1772-1779.

9. Attur MG, Dave MN, Clancy RM, Patel IR, Abramson SB, Amin AR: Functional genomic analysis in arthritis-affected cartilage: yin-yang regulation of inflammatory mediators by alpha 5 beta 1 and alpha V beta 3 integrins. J Immunol 2000, 164:2684-2691.
10. Postigo AA, Garcia-Vicuna R, Laffon A, Sanchez-Madrid F: The role of adhesion molecules in the pathogenesis of rheumatoid arthritis. Autoimmunity 1993, 16:69-76.

11. Liao HX, Haynes BF: Role of adhesion molecules in the pathogenesis of rheumatoid arthritis. Rheum Dis Clin North Am 1995, 21:715-740.

12. Laudanna C, Kim JY, Constantin G, Butcher E: Rapid leukocyte integrin activation by chemokines. Immunol Rev 2002, 186:37-46.

13. Yee $K L$, Weaver VM, Hammer DA: Integrin-mediated signalling through the MAP-kinase pathway. IET Syst Bio/ 2008, 2:8-15.

14. Zutter MM, Santoro SA, Staatz WD, Tsung YL: Re-expression of the alpha 2 beta 1 integrin abrogates the malignant phenotype of breast carcinoma cells. Proc Natl Acad Sci U S A 1995, 92:7411-7415.

15. Rinaldi N, Barth T, Henne C, Mechterscheimer G, Moller P: Synoviocytes in chronic synovitis in situ and cytokine stimulated synovial cells in vitro neo-express alpha 1, alpha 3 and alpha 5 chains of beta 1 integrins. Virchows Arch 1994, 425:171-180.

16. Johnson BA, Haines GK, Harlow LA, Koch AE: Adhesion molecule expression in human synovial tissue. Arthritis Rheum 1993, 36:137-146.

17. Rinaldi N, Weis D, Brado B, Schwarz-Eywill M, Lukoschek M, Pezzutto A, Keilholz U, Barth TF: Differential expression and functional behaviour of the alpha $v$ and beta 3 integrin subunits in cytokine stimulated fibroblast-like cells derived from synovial tissue of rheumatoid arthritis and osteoarthritis in vitro. Ann Rheum Dis 1997, 56:729-736.

18. Pirila $L$, Aho H, Roivainen A, Konttinen YT, Pelliniemi LJ, Heino J: Identification of alpha6beta1 integrin positive cells in synovial lining layer as type B synoviocytes. J Rheumatol 2001, 28:478-484.

19. Pfaff M, Aumailley M, Specks U, Knolle J, Zerwes HG, Timpl R: Integrin and Arg-Gly-Asp dependence of cell adhesion to the native and unfolded triple helix of collagen type VI. Exp Cell Res 1993, 206:167-176.

20. Davis GE: Affinity of integrins for damaged extracellular matrix: alpha $v$ beta 3 binds to denatured collagen type I through RGD sites. Biochem Biophys Res Commun 1992, 182:1025-1031.

21. Rubio MA, Sotillos M, Jochems G, Alvarez V, Corbi AL: Monocyte activation: rapid induction of alpha 1/beta 1 (VLA-1) integrin expression by lipopolysaccharide and interferon-gamma. Eur J Immunol 1995, 25:2701-2705

22. Takahashi H, Soderstrom K, Nilsson E, Kiessling R, Patarroyo M: Integrins and other adhesion molecules on lymphocytes from synovial fluid and peripheral blood of rheumatoid arthritis patients. Eur J Immunol 1992, 22:2879-2885

23. Senger DR, Claffey KP, Benes JE, Perruzzi CA, Sergiou AP, Detmar M: Angiogenesis promoted by vascular endothelial growth factor: regulation through alpha1 beta1 and alpha2beta1 integrins. Proc Natl Acad Sci U SA 1997, 94:13612-13617.

24. Boisvert M, Gendron S, Chetoui N, Aoudjit F: Alpha2 beta1 integrin signaling augments $T$ cell receptor-dependent production of interferon-gamma in human T cells. Mol Immunol 2007, 44:3732-3740.

25. de Fougerolles AR, Sprague AG, Nickerson-Nutter CL, Chi-Rosso G, Rennert PD, Gardner H, Gotwals PJ, Lobb RR, Koteliansky VE: Regulation of inflammation by collagen-binding integrins alpha1beta 1 and alpha2beta1 in models of hypersensitivity and arthritis. J Clin Invest 2000, 105:721-729.

26. Boisvert M, Chetoui N, Gendron S, Aoudjit F: Alpha2beta1 integrin is the major collagen-binding integrin expressed on human Th17 cells. Eur J Immunol 2010, 40:2710-2719.

27. Miner JH: Laminins and their roles in mammals. Microsc Res Tech 2008, 71:349-356.

28. Schneider M, Voss B, Rauterberg J, Menke M, Pauly T, Miehlke RK, Friemann J, Gerlach U: Basement membrane proteins in synovial membrane: distribution in rheumatoid arthritis and synthesis by fibroblast-like cells. Clin Rheumatol 1994, 13:90-97.

29. Hoberg M, Rudert M, Pap T, Klein G, Gay S, Aicher WK: Attachment to laminin-111 facilitates transforming growth factor beta-induced expression of matrix metalloproteinase-3 in synovial fibroblasts. Ann Rheum Dis 2007, 66:446-451.

30. Pankov R, Yamada KM: Fibronectin at a glance. J Cell Sci 2002, 115:3861-3863.

31. Yasuda T: Cartilage destruction by matrix degradation products. Mod Rheumatol 2006, 16:197-205.

32. Davis LS, Oppenheimer-Marks N, Bednarczyk JL, McIntyre BW, Lipsky PE. Fibronectin promotes proliferation of naive and memory $T$ cells by signaling through both the VLA-4 and VLA-5 integrin molecules. J Immuno/ 
1990, 145:785-793.

33. Kitagawa A, Miura Y, Saura R, Mitani M, Ishikawa H, Hashiramoto A, Yoshiya S, Shiozawa S, Kurosaka M: Anchorage on fibronectin via VLA-5 (alpha5beta1 integrin) protects rheumatoid synovial cells from Fas-induced apoptosis. Ann Rheum Dis 2006, 65:721-727.

34. Zeisel MB, Druet VA, Wachsmann D, Sibilia J: MMP-3 expression and release by rheumatoid arthritis fibroblast-like synoviocytes induced with a bacterial ligand of integrin alpha5beta1. Arthritis Res Ther 2005 7:R118-R126.

35. Alsaleh G, Francois A, Knapp AM, Schickel JN, Sibilia J, Pasquali JL, Gottenberg JE, Wachsmann D, Soulas-Sprauel P: Synovial fibroblasts promote immunoglobulin class switching by a mechanism involving BAFF. Eur J Immunol 2011, 41:2113-2122.

36. Hyun YM, Chung HL, McGrath JL, Waugh RE, Kim M: Activated integrin VLA-4 localizes to the lamellipodia and mediates T cell migration on VCAM-1. J Immunol 2009, 183:359-369.

37. Raychaudhuri A, Chou M, Weetall M, Jeng AY: Blockade of integrin VLA-4 prevents inflammation and matrix metalloproteinase expression in a murine model of accelerated collagen-induced arthritis. Inflammation 2003, 27:107-113.

38. Jacq L, Garnier S, Dieudé P, Michou L, Pierlot C, Migliorini P, Balsa A, Westhovens R, Barrera P, Alves H, Vaz C, Fernandes M, Pascual-Salcedo D, Bombardieri S, Dequeker J, Radstake TR, Van Riel P, van de Putte L, Lopes-Vaz A, Glikmans E, Barbet S, Lasbleiz S, Lemaire I, Quillet P, Hilliquin P, Teixeira VH, Petit-Teixeira E, Mbarek H, Prum B, Bardin T, et al.: The ITGAV rs3738919-C allele is associated with rheumatoid arthritis in the European Caucasian population: a family-based study. Arthritis Res Ther 2007, 9:R63.

39. Charo IF, Nannizzi L, Smith JW, Cheresh DA: The vitronectin receptor alpha $v$ beta 3 binds fibronectin and acts in concert with alpha 5 beta 1 in promoting cellular attachment and spreading on fibronectin. $J$ Cell Biol 1990, 111:2795-2800.

40. Nakamura I, Duong le T, Rodan SB, Rodan GA: Involvement of alpha(v)beta3 integrins in osteoclast function. J Bone Miner Metab 2007, 25:337-344.

41. Wilder RL: Integrin alpha V beta 3 as a target for treatment of rheumatoid arthritis and related rheumatic diseases. Ann Rheum Dis 2002, 61 Suppl 2:ii96-ii99.
42. Alghisi GC, Ponsonnet $\mathrm{L}$, Ruegg C: The integrin antagonist cilengitide activates alphaVbeta3, disrupts VE-cadherin localization at cell junctions and enhances permeability in endothelial cells. PLoS One 2009, 4:e4449.

43. Neumann E, Gay S, Muller-Ladner U: The RANK/RANKL/osteoprotegerin system in rheumatoid arthritis: new insights from animal models. Arthritis Rheum 2005, 52:2960-2967.

44. Lindsley HB, Smith DD, Cohick CB, Koch AE, Davis LS: Proinflammatory cytokines enhance human synoviocyte expression of functional intercellular adhesion molecule-1 (ICAM-1). Clin Immunol Immunopathol 1993, 68:311-320

45. Jasin HE, Lightfoot E, Davis LS, Rothlein R, Faanes RB, Lipsky PE: Amelioration of antigen-induced arthritis in rabbits treated with monoclonal antibodies to leukocyte adhesion molecules. Arthritis Rheum 1992, 35:541-549.

46. Suchard SJ, Stetsko DK, Davis PM, Skala S, Potin D, Launay M, Dhar TG, Barrish JC, Susulic V, Shuster DJ, McIntyre KW, McKinnon M, Salter-Cid L: An LFA-1 (alphaLbeta2) small-molecule antagonist reduces inflammation and joint destruction in murine models of arthritis. J Immunol 2010, 184:3917-3926.

47. Major EO: Progressive multifocal leukoencephalopathy in patients on immunomodulatory therapies. Annu Rev Med 2010, 61:35-47.

48. Bullard DC, Hurley LA, Lorenzo I, Sly LM, Beaudet AL, Staite ND: Reduced susceptibility to collagen-induced arthritis in mice deficient in intercellular adhesion molecule-1. J Immunol 1996, 157:3153-3158.

49. Kavanaugh AF, Davis LS, Jain RI, Nichols LA, Norris SH, Lipsky PE: A phase I/II open label study of the safety and efficacy of an anti-ICAM-1 (intercellular adhesion molecule-1; CD54) monoclonal antibody in early rheumatoid arthritis. J Rheumatol 1996, 23:1338-1344.

50. Vuorte J, Lindsberg PJ, Kaste M, Meri S, Jansson SE, Rothlein R, Repo H: AntiICAM-1 monoclonal antibody R6.5 (Enlimomab) promotes activation of neutrophils in whole blood. J Immunol 1999, 162:2353-2357.

doi:10.1186/ar3464

Cite this article as: Lowin T, Straub RH: Integrins and their ligands in rheumatoid arthritis. Arthritis Research \& Therapy 2011, 13:244. 